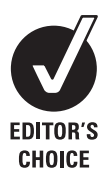

${ }^{1}$ Harvard School of Public Health, Boston, Massachusetts, USA; ${ }^{2}$ Institute for Healthcare Improvement, Cambridge, Massachusetts, USA; ${ }^{3}$ Agency for Healthcare Research and Quality, Bethesda, Maryland, USA; ${ }^{4}$ National Patient Safety Foundation, Boston,

Massachusetts, USA:

${ }^{5}$ Consumers Union, Yonkers, New York, USA; ${ }^{6}$ Kaiser

Foundation Health Plan (retired), Oakland, California, USA;

${ }^{7}$ Vanderbilt University Medical Center, Nashville, Tennessee, USA; ${ }^{8}$ The Joint Commission (retired), USA; ${ }^{9}$ Alcoa (retired), Pittsburgh, Pennsylvania, USA;

${ }^{10}$ Dana-Farber Cancer Institute, Boston, Massachusetts, USA

Dr L L Leape, Harvard School of Public Health, 677 Huntington Avenue, Boston, MA 02115,

USA; leape@hsph.harvard.edu

Accepted 13 October 2009

\title{
Transforming healthcare: a safety imperative
}

\author{
L Leape, ${ }^{1}$ D Berwick, ${ }^{1,2}$ C Clancy, ${ }^{3} \mathrm{~J}$ Conway, ${ }^{2}$ P Gluck, ${ }^{4} \mathrm{~J} \mathrm{Guest}^{5}$ D Lawrence, ${ }^{6}$ \\ J Morath, ${ }^{7}$ D O'Leary, ${ }^{8}$ P O'Neill, ${ }^{9}$ D Pinakiewicz, ${ }^{4} \mathrm{~T}$ Isaac, ${ }^{10}$ for the Lucian Leape \\ Institute at the National Patient Safety Foundation
}

\section{ABSTRACT}

Ten years ago, the Institute of Medicine reported alarming data on the scope and impact of medical errors in the US and called for national efforts to address this problem. While efforts to improve patient safety have proliferated during the past decade, progress toward improvement has been frustratingly slow. Some of this lack of progress may be attributable to the persistence of a medical ethos, institutionalized in the hierarchical structure of academic medicine and healthcare organizations, that discourages teamwork and transparency and undermines the establishment of clear systems of accountability for safe care. The Lucian Leape Institute, established by the US National Patient Safety Foundation to provide vision and strategic direction for the patient safety work, has identified five concepts as fundamental to the endeavor of achieving meaningful improvement in healthcare system safety. These five concepts are transparency, care integration, patient/consumer engagement, restoration of joy and meaning in work, and medical education reform. This paper introduces the five concepts and illustrates the meaning and implications of each as a component of a vision for healthcare safety improvement. In future roundtable sessions, the Institute will further elaborate on the meaning of each concept, identify the challenges to implementation, and issue recommendations for policy makers, organizations, and healthcare professionals.

Healthcare is unsafe. In its groundbreaking report, To Err Is Human, the Institute of Medicine (IOM) estimated that, in the USA, as many as a million people were injured and 98000 died annually as a result of medical errors. ${ }^{1}$ Subsequent studies in multiple countries suggest these may be underestimates. $^{2-5}$ The IOM called in 2000 for a major national effort to reduce medical errors by $50 \%$ within 5 years, ${ }^{1}$ but progress since has fallen far short. ${ }^{6-8}$ Many patients continue to fear, justifiably, that they may be harmed when they enter a hospital.

The slow progress is not for want of trying. Both public and private organisations have initiated major programmes to develop and implement new safe practices and to train healthcare workers in patient safety. ${ }^{9-16}$ In the USA, since 1997, the National Patient Safety Foundation has worked with stakeholder groups to advance learning and bring forward new solutions. The Agency for Healthcare Research and Quality has invested in defining measures to assess and improve safety and to build capacity through its Patient Safety Improvement Corps. ${ }^{17}$ The National Quality Forum has certified safe practices ready for use. ${ }^{18}$ The Joint Commission has required hospital compliance with new patient safety goals. ${ }^{19}$ The
Institute for Healthcare Improvement has launched two massive national and international campaigns ${ }^{11}$ to inspire thousands of hospitals to adopt evidence-based safe practices.

Similar advances have occurred in many other countries. Voluntary nongovernmental patient safety organisations have been established in Denmark, Canada, Spain, Sweden, and Switzerland. Many have conducted studies to determine the extent of medical injury, and several have developed reporting systems. ${ }^{20}{ }^{21}$ In Australia, the work of the Australian Council on Safety and Quality continued when the Australian Commission of Safety and Quality in Health Care was established by the government to develop a national strategic framework and associated patient safety work programme.

The UK has led the way in government commitment to safety, with the establishment of the National Patient Safety Agency under the Department of Health, and has developed a reporting system and a clinical assessment service. The department has also established and enforced performance measures. In addition, voluntary efforts, such as the Patient Safety First campaign, have been extensive. Liam Donaldson from the National Health Service also led the formation of the World Alliance for Patient Safety, which has launched seven major programmes, including successful worldwide hand hygiene and surgical checklist campaigns. ${ }^{22} 23$

However, these efforts have been insufficient. As other industries have learned, safety does not depend just on measurement, practices and rules, nor does it depend on any specific improvement methods; it depends on achieving a culture of trust, reporting, transparency and discipline. For healthcare organisations in every country, this requires major culture change.

Too many healthcare organisations fit James Reason's definition of the "sick system syndrome." They are hierarchical and deficient in mutual respect, teamwork and transparency. Blame is still a mainstay solution. Mechanisms for ensuring accountability are weak and ambiguous. Few have the capacity to learn and change that is characteristic of the so-called high reliability industries. ${ }^{24}$ Most do not recognise that safety should be a precondition, not a priority. Or that fulfilling the interests of their patients in safe care and of their staffs in a safe workplace will enhance productivity and profitability.

Many physicians do not know how to be team players and regard other health workers as assistants. Outmoded hierarchical structures inhibit 
collaboration and learning. Nurses are trapped in rigid organisational structures in which they often spend more time tending to their records than to their patients. Often, their work environment does not permit them to realise their full potential and is unsafe because of system vulnerabilities and leadership inattention. Too many practitioners-doctors, nurses, pharmacists, therapists, technicians-function in "silos," focusing on their own performance and communicating with others in fragmented and inefficient ways that inhibit teamwork. Patients are seldom included in organisational planning or in the analysis of adverse events that have harmed them. ${ }^{25} 26$

\section{WHAT NEEDS TO BE DONE?}

The Lucian Leape Institute was established by the National Patient Safety Foundation to provide strategic guidance for achieving safe healthcare. Like the vast majority of safety experts, we believe that healthcare entities must become "highreliability organisations" that hold themselves accountable to consistently offer safe, effective, patient-centred care. ${ }^{24}$ This will require all parties-hospitals and their boards, doctors, nurses, pharmacists, administrators, regulators, government officials, payers, professional societies, and patients-to move beyond the IOM recommendations for changes in systems and to radically change the ways in which they think about care and how it is provided. Healthcare needs not just to be improved but to be transformed.

\section{A VISION FOR TRANSFORMATION}

We envision a culture that is open, transparent, supportive and committed to learning; where doctors, nurses and all health workers treat each other and their patients competently and with respect; where the patient's interest is always paramount; and where patients and families are fully engaged in their care. We envision a culture centred on teamwork, grounded in mission and purpose, in which organisational managers and boards hold themselves accountable for safety and learning to improve. In a learning organisation, every voice is heard and every worker is empowered to prevent system breakdowns and correct them when they occur. The culture we envision aspires to, strives for, and achieves unprecedented levels of safety, effectiveness, and satisfaction in healthcare.

How do we get there? We believe that to become safe, effective, high reliability organisations, healthcare organisations must implement five major transforming concepts. Although many other ideas and actions are needed to bring about the changes needed in our complex system, we believe these are the essential core: if an organisation achieves them all, it will be well on the way to becoming a high reliability organisation. If not, it is unlikely to succeed.

The five transforming concepts are as follows: (1) transparency must be a practiced value in everything we do; (2) care must be delivered by multidisciplinary teams working in integrated care platforms; (3) patients must become full partners in all aspects of healthcare; (4) healthcare workers

\section{A vision for healthcare}

We envision a culture that is open, transparent, supportive and committed to learning; where doctors, nurses and all health workers treat each other and their patients competently and with respect; where the patient's interest is always paramount; and where patients and families are fully engaged in their care. need to find joy and meaning in their work; and (5) medical education must be redesigned to prepare new physicians to function in this new environment.

Each of these concepts calls for moving thinking beyond current boundaries and each implies profound behavioural changes. We will develop these ideas further in stakeholder roundtables for each concept that will define the challenges in detail and make specific recommendations to policy makers, organisations and healthcare professionals.

\section{TRANSPARENCY}

Transparency - the free, uninhibited sharing of information - is probably the most important single attribute of a culture of safety. In complex, tightly coupled systems like healthcare, transparency is a precondition to safety. Its absence inhibits learning from mistakes, distorts collegiality and erodes patient trust.

Healthcare leaders have been far too timid about becoming truly transparent. We urge giant steps-now. Healthcare organisations must become transparent in all dimensions: among caregivers, between caregivers and patients, between organisations, and with the public.

First, caregivers need to share information openly about hazards, errors and adverse events. People cannot improve systems if they cannot talk about what they are experiencing. Individuals must be able to report errors without fear of punishment or embarrassment. They must be convinced that the response will be, not, "Who failed?" but, rather, "What happened?"

Second, caregivers need to be open with patients when things go wrong. Unfortunately, many risk managers still coach clinicians to limit what they reveal, blaming the malpractice dragon, despite examples, such as the University of Michigan Hospital, that have adopted "extreme honesty" and seen substantial decreases in the number of suits and costs. ${ }^{27} \mathrm{We}$ should emulate their bold example: promptly acknowledge when things go wrong, explain the causes as they are understood and apologise when patient harm comes from failures in care. Hospital leaders must fully support caregivers as they strive to be more transparent.

This form of transparency is not just a technical imperative, it is a moral imperative. We have neither a legal nor a moral right to withhold from patients information on harm done to them, even if that harm is accidental.

Third, just as individual clinicians should exchange information on injuries and hazards, so should organisations. In the aviation industry, if a hydraulic device proves faulty in Dallas, the sun will not set before mechanics know about it in Denver and Dubai. However, in healthcare, organisations hesitate to exchange lessons openly for many of the same reasons that individual staff do. To make this sharing worthwhile, healthcare organisations also need to invest heavily in the analysis of those reports by experienced professionals.

\section{Five transforming concepts}

Transparency

- Integrated care platform

- Consumer engagement

- Joy and meaning in work

- Medical education reform 
The fourth meaning of "transparency" is the one that most laypeople, purchasers and regulators use: public reporting about harmful incidents. Many organisations have championed public reporting on harm, and some states are now requiring it for socalled never events.

So far, healthcare has addressed transparency mainly in the form of incident-reporting systems-our fourth definition. A more robust approach will serve us better: extreme transparency of all four types: among staff, between caregivers and patients, among institutions, and in open and clear reports to the public at large.

\section{INTEGRATED CARE PLATFORMS}

The integrated care platform is an organisational structure within a healthcare system that enhances quality and patient safety by bringing together across all venues-inpatient, outpatient and residential - the care and the support systems required to provide evidence-based, appropriate and responsive care to patients according to their needs (such as various chronic diseases). ${ }^{28}$

The purpose of the platform is to maximise efficiency, safety, quality and reliability to produce consistently superior outcomes at the lowest cost. It fosters the multidisciplinary solutions that are essential for safe management of complex clinical conditions. Distinct platforms are designed for conditions that share common work and support requirements, such as chronic disease care, complex acute care, palliative and endof-life care.

Every care platform must have the following characteristics:

- Patient centredness: personnel, facilities and services are organised to meet all patients' needs efficiently and responsively; to be available when and where needed, 24/ 7; and to include the patient and family as partners in care.

- Work assignment: work is assigned to the individuals who are responsible for its completion. Assignments strive to maximise the performance capability of each individual while ensuring that work is done by the least expensive qualified caregiver or multidisciplinary team at the location most accessible to the patient. The physician participates when his/her special expertise is required and when patient expectations permit no alternative.

- Support: The support framework-people, systems and tools (eg, technologies, IT, telecommunications) - is defined by the work and patient participation design.

- Community linkage: Linkages to community advocacy, support, and education groups (especially health literacy) are incorporated into the design as appropriate (eg, for patients with chronic conditions).

- Variation management: Ensuring quality and efficiency requires determining whether variations in process are appropriate (ie, evidence-based). Exception analysis assesses whether variations result from (1) adaptations to a specific patient requirement, (2) evolution of new evidence (good), (3) lack of training in appropriate care or (4) poorly defined care pathways (bad).

- Transparency: Because care is designed and expected variation is defined, both the output and delivery process within a platform can be observed, measured and shared with all concerned, including patients.

Dividing healthcare needs into disease or condition groupings and designing an integrated care platform for each achieves the impact lacking in other integration approaches. It also places accountability at the appropriate level-the integrated system-rather than solely on the individual clinician.

\section{CONSUMER ENGAGEMENT_-"NOTHING ABOUT ME WITHOUT} ME"

The engagement of consumers in care partnerships is essential to achieve quality and safety in healthcare. ${ }^{25}$ Whether pursuing healthy living, as patients receiving care, or as purchasers (future patients), individuals and their families must play a central role. The guiding principle is "If health is on the table, then the patient and family must be at the table, every table, now."

In 2001, the IOM report "Crossing the Quality Chasm" included patient centredness as one of the six core aims for healthcare. ${ }^{29}$ Earlier, in 1997, the Salzburg Seminar suggested that efforts to improve care might take strikingly different shape if patients worked as full partners with caregivers to design and implement change. The patient experience should be "nothing about me, without me." 30

The power of the involvement of patients and families is seen in their contributions to the safety system, in recognising and responding to literacy problems, in the improved management of acute and chronic diseases and in sharing experiences so that others can learn. ${ }^{31} 32$

Despite the evidence of the effectiveness of consumer engagement, ${ }^{33}{ }^{34}$ implementation to date has been modest. Actions are more often for than with the consumer. Many clinicians are reluctant to share knowledge and care plans with patients. Analysis of safety systems and adverse events has not usually involved patients, even in areas where they have a great deal to add, such as medication management and transitions in care. Consumer advocacy groups have not always been welcomed as participants in organisational and community policy-setting efforts.

We envisage patients as essential and respected partners in their own care and in the design and execution of all aspects of healthcare. In this new world of healthcare:

- Organisations publicly and consistently affirm the centrality of patient- and family-centred care. They seek out patients, listen to them, hear their stories, are open and honest with them, and take action with them.

- The family is respected as part of the care team-never visitors-in every area of the hospital, including the emergency department and the intensive care unit.

- Patients share fully in decision-making and are guided on how to self-manage, partner with their clinicians and develop their own care plans. They are spoken to in a way they can understand and are empowered to be in control of their care.

\section{JOY AND MEANING IN WORK}

Caregivers cannot meet the challenge of making healthcare safe unless they feel valued and find joy and meaning in their work. The evidence abounds that in the USA, many do not. In a recent survey, $60 \%$ of physicians indicated they were considering leaving medical practice because they are discouraged ${ }^{35}$; a study of newly licensed registered nurses showed that 33\% might seek another job within the year. ${ }^{36}$

Among physicians, reasons include loss of control, the malpractice liability threat and declining revenues. ${ }^{37}$ Among nurses, lack of respect from both administrators and physicians ranks high, along with the increasing burden of regulation and record-keeping that separates them from patient care. For many, the transformation of healthcare from a public service to a business in the last quarter of the 20th century reduced complex, highly intimate care processes to transactional industrial production schemata, divorcing work from meaning. 
Another cause of poor morale is tolerance of disrespectful and disruptive behaviour. Sixty-two per cent of nurses reported verbal abuse as the most frequently encountered injury at work. ${ }^{38} \mathrm{~A}$ permissive environment exacerbates the risk-prone conditions in which people work, demoralises workers and leads to conflict. ${ }^{39} 40$ Failure of leadership to address interpersonal communication issues depletes the energy of an organisation and raises doubt about the organisation's commitment to fairness.

Although addressing some of these issues requires major national policy changes, it is also a fact that some healthcare organisations have created environments where morale is high and workers do find joy and meaning in their work. This strongly suggests that the causes-and the remedies-are local. Creating an environment where every worker finds joy and meaning in work is a foundational leadership challenge for a healthcare organisation.

What needs to be done? Capturing the soul of an organisation, where joy and meaning resides, requires a true partnership to align values among organisation leaders, professionals and the workforce. Leaders must create the environment where it is possible for improvements to take place. However, the richest source of ideas for improvement is the frontline workers. It is they who live in the complexities of the current systems, have direct insights into failures and see daily opportunities for improvement. ${ }^{41}$

These lessons can only be harvested if all members of the workforce feel valued and work together in meaningful teams. This requires that everyone is (a) treated with dignity and respect; (b) given the education, training, tools and encouragement they need to make a contribution that gives meaning to their life; and (c) recognised and appreciated for what they do. ${ }^{42}$

Leaders have a choice: they can view organisations as industrial models and focus on restructuring, production and regulation, or they can, as we urge, view them as being composed of people with the skills and energy to perform meaningful work, and focus on the shared vision and values that provide meaning and joy in work.

\section{REFORM OF MEDICAL EDUCATION}

Medical education needs to be restructured to reduce its almost exclusive focus on the acquisition of scientific and clinical facts and to emphasise the development of skills, behaviours and attitudes needed by practicing physicians. These include the ability to manage information; understanding of the basic concepts of human interaction, patient safety, healthcare quality and systems theory; and possession of management, communication and teamwork skills. Although a similar need exists across all health professions, it is most compelling in medicine because the decisions of physicians influence the care that all other professionals provide.

The principal conclusion of the To Err Is Human report is that the major cause of adverse events is poorly designed systems, not negligent individual performance. ${ }^{1}$ The implication is that physicians, managers, nurses and others should work together in teams to redesign flawed processes to prevent harm. One reason this has not happened faster is that physicians have not been educated to carry out this critically important work.

In the typical medical school curriculum, little or no instruction is provided in engineering concepts applicable to systems thinking, safety science, improvement science, human factors, leadership or teamwork. Students obtain little experience in examining the patient care processes, which constitute the everyday practice in the real world of healthcare or experience working with students in nursing, pharmacy or other health fields. Nor do they receive instruction in skills needed to communicate effectively with coworkers and patients, or how to deal with their own feelings of doubt, fear and uncertainty. Yet, these are the knowledge and skills that most people consider essential for a physician.

Over the past 5 years, the IOM, ${ }^{43}$ the Accreditation Council for Graduate Medical Education ${ }^{44}$ and the American Board of Medical Specialties ${ }^{45}$ have formulated concise sets of desired practitioner behavioural competencies. These suggest that medical schools should pay greater attention to teaching concepts that underlie the behaviours for which future physicians will be held accountable. That teaching should be undertaken in an interdisciplinary fashion and capitalise on the rapidly expanding applications of simulation as a teaching tool.

Today's medical schools are producing square pegs for our care system's round holes. This disconnect requires immediate attention, as does the need for retraining practicing physicians, who are the students' mentors and role models.

\section{CONCLUSION}

These transformations comprise a major culture change for healthcare. Achieving them will require enlightened leadership, commitment and support from all stakeholders. However, without them, we believe progress in making healthcare safe will continue to sputter.

Competing interests: None.

\section{REFERENCES}

1. Kohn KT, Corrigan JM, Donaldson MS, eds. To err is human: Building a safer health system. Washington, DC: National Academy Press, 1999.

2. Vincent C, Neale G, Woloshynowych M. Adverse events in British hospitals: preliminary retrospective record review. BMJ 2001;322:517-9.

3. Wilson R, Runciman W, Gibberd R, et al. The quality in Australian health care study. Med J Aust 1995;163:458-71.

4. Baker GR, Norton PG, Flintoft V, et al. The Canadian Adverse Events Study: the incidence of adverse events among hospital patients in Canada. CMAJ 2004;170:1678-86.

5. Davis P, Lay-Yee R, Briant R, et al. Adverse events in New Zealand public hospitals: Principal findings from a national survey. Wellington (New Zealand): Ministry of Health, 2001 Dec, Occasional Paper 3.

6. Kwaan MR, Studdert DM, Zinner MJ, et al. Incidence, patterns, and prevention of wrong-site surgery. Arch Surg 2006;141:353-7; discussion 357-8.

7. AHRQ. 2004 National Healthcare Quality Report. Rockville, MD: AHRO, 2004 Dec.

8. Pronovost $\mathbf{P}$, Miller MR, Wachter RM. Tracking progress in patient safety-an elusive target. JAMA 2006;296:696-9.

9. Hendrich A, Tersigni AR, Jeffcoat $S$, et al. The Ascension Health journey to zero: lessons learned and leadership. Jt Comm J Qual Patient Saf 2007;33:739-49.

10. McCannon CJ, Hackbarth AD, Griffin FA. Miles to go: an introduction to the 5 Million Lives Campaign. Jt Comm J Qual Patient Saf 2007;33:477-84.

11. IHI. http://www.ihi.org//HI/Programs/Campaign/ (accessed 3 November 2009).

12. IHI. The breakthrough series: IHI's collaborative model for achieving breakthrough improvement. Diabetes Spectrum 2004;17:97-101.

13. Mann SM, Marcus R, Sachs B. Lessons from the cockpit: how team training can reduce errors on L\&D. Contemporary Ob/GYN 2006;51:34-45.

14. Kuperman GJ, Gibson RF. Computer physician order entry: benefits, costs, and issues. Ann Intern Med 2003;139:31-9.

15. Friedrich MJ. Practice makes perfect: risk-free medical training with patient simulators. JAMA 2002;288:2808-12.

16. Bagian JP, Lee C, Gosbee J, et al. Developing and deploying a patient safety program in a large health care delivery system: you can't fix what you don't know about. Jt Comm J Qual Patient Saf 2001;27:522-32.

17. AHRO. Quality indicators. 2004. http://www.qualityindicators.ahrq.gov (accessed 18 Dec 2004)

18. National Quality Forum. Safe practices for better health care: a consensus report Washington (DC): NQF, 2003, NQFCR-05-03.

19. Joint Commission. 2009 Patient safety goals. http://www.jointcommission.org/ PatientSafety/NationalPatientSafetyGoals (accessed 23 Sept 2008). 
20. Runciman WB. Lessons from the Australian Patient Safety Foundation: setting up a national patient safety surveillance system - is this the right model? Qual Saf Health Care 2002;11:246-51.

21. NBP. Can the NPSA collect useful data about adverse patient safety incidents electronically? - a report on the pilot data audit undertaken by the NPSA. 2003/04. National Patient Safety Agency. Pilot Project Evaluation Report. http://www.ncas. npsa.nhs.uk/EasySiteWeb/getresource.axd?AssetID =2652\&type =full\&servicetype $=$ Attachment (accessed 5 Nov 2009).

22. Haynes $\mathbf{A B}$, Weiser TG, Berry WR, et al. A surgical safety checklist to reduce morbidity and morality in a global population. N Engl J Med 2009;360:491-9.

23. WAPS. Global patient safety challenge. http://www.who.int/gpsc/en (accessed 30 Oct 2007).

24. Weick KE, Sutcliffe KM, Obstfeld D. Organizing for high reliability. Res Org Behav 1999;21:81-123.

25. Conway J, Johnson B, Edgman-Levitan S, et al. Partnering with patients and families to design a patient- and family-centered health care system: a roadmap for the future. Institute for Family-Centered Care and Institute for Healthcare Improvement. http://www.ihi.org//HI/Topics/PatientCenteredCare/PatientCenteredCareGeneral/ Literature/PartneringwithPatientsandFamilies.htm (accessed 8 Jul 2008)

26. Walshe K, Shortell SM. When things go wrong: how health care organizations deal with major failures. Health Aff (Millwood) 2004;23:103-11.

27. Boothman RC. Apologies and a strong defense at the University of Michigan Health System. Physician Exec 2006 Mar/Apr:7-10

28. Bohmer R, Lawrence D. Care platforms: a basic building block for care delivery. Health Aff 2008:27:1336-40.

29. Institute of Medicine. Crossing the quality chasm. Washington (DC): National Academy Press, 2001.

30. Delbanco T, Berwick D, Boufford J, et al. Healthcare in a land called PeoplePower: nothing about me without me. Health Expect 2001;4:144-50.

31. Institute for Family Centered Care. Bibliography and other resource materials for advancing patient- and family-centered care. http://www.familycenteredcare.org/ advance/IFCC Bibliography.pdf (accessed 4 Feb 2008).

32. Johnson B, A braham M, Conway J, et al. Partnering with patients and families to design a patient and family-centered health care system: recommendations and promising practices. Institute for Family-Centered Care and Institute for Healthcare Improvement. http://www.familycenteredcare.org/pdf/PartneringwithPatients.pdf (accessed 8 Jul 2008).

33. Robert Wood Johnson Foundation. Improving quality health care: the role of consumer engagement. http://www.rwjf.org/programareas/resources/product. jsp?id=23071\&pid=1142 (accessed 1 Feb 2008).

34. Partnership for Healthcare Excellence. Research review: public opinion on healthcare quality. http://www.partnershipforhealthcare.org/newsroom/ ConsumerResearchReview.ppt (accessed 1 Feb 2008).

35. American College of Physician Executives. "Physician Morale Survey" J Med Manage 2006;32:6-15

36. Kovener CT, Brewer CS, Fairchild S, et al. Newly licensed RN's characteristics, attitudes, and intentions to work. Am J Nurs 2007:107:58-70.

37. Spickard A Jr, Gabbe SG, Christensen JF. Mid-career burnout in generalist and specialist physicians. JAMA 2002;288:1447-50.

38. Rosenstein $\mathbf{A H}$, Russell H, Lauve R. Disruptive physician behavior contributes to nursing shortage. Study links bad behavior by doctors to nurses leaving the profession. Physician Exec 2002;28:8-11.

39. Benzer DG, Miller MM. The disruptive-abusive physician: a new look at an old problem. Wis Med J 1995;94:455-60.

40. Diaz AL, McMillin JD. A definition and description of nurse abuse. West J Nurs Res 1991;13:97-109.

41. Kockhan T, McKersie R, Eaton A, et al. The Kaiser Permanente Labor Management Partnership: 2002-2004. Cambridge (MA): MIT Institute for Work and Employment Research, MIT Sloan School of Management, 2005 May.
42. O'Neill P. Foreword. In: Cox T, ed. Creating the multicultural organization. New York: Jossey-Bass, 2001

43. Greiner AC, Knebel E. Health professions education: a bridge to quality. Washington (DC): National Academy Press, 2003.

44. ACGME. ACGME common program requirements: general competencies. http:// www.acgme.org/outcome/comp/GeneralCompetenciesStandards21307.pdf (accessed 14 Sept 2008).

45. ABMS. ABMS maintenance of certification: MOC competencies and criteria. http:// www.abms.org/Maintenance of Certification/ABMS MOC.aspx laccessed 14 Sept 2008).

\section{APPENDIX}

The Lucian Leape Institute at the National Patient Safety Foundation

Lucian L. Leape, MD, leape@hsph.harvard.edu

Chair, Lucian Leape Institute at NPSF

Adjunct Professor of Health Policy

Harvard School of Public Health

Diane C. Pinakiewicz, MBA, dpinakiewicz@npsf.org

President, Lucian Leape Institute at NPSF

President

National Patient Safety Foundation

Donald M, Berwick, MD, MPP, dberwick@ihi.org

President and Chief Executive Officer

Institute for Healthcare Improvement

Carolyn M. Clancy, MD, carolyn.clancy@ahrq.hhs.gov

Director

Agency for Healthcare Research and Quality

James B. Conway, MAM, CHE, jconway@ihi.org

Senior Vice President

Institute for Health Care Improvement

James Guest, JD, jguest@consumer.org

President

Consumer Union

David Lawrence, MD, dmlawrencemd@gmail.com

Chairman and CEO (retired)

Kaiser Foundation Health Plan and

Kaiser Foundation Hospitals

Julianne M. Morath, RN, BS, julie.morath@childrensmn.org

Chief Operating Officer

Children's Hospitals and Clinics of Minnesota

Dennis S. O’Leary, MD, do'leary@jointcommission.org

President Emeritus

The Joint Commission

Paul O’Neill, poneillpa@aol.com

Former Chairman and CEO: Alcoa

72nd Secretary of the US Treasury

Ex-Officio

Paul A. Gluck, MD, astrogld2@aol.com

Immediate Past Chair

NPSF Board of Directors

Thomas Isaac, MD, txi001@gmail.com

Institute Fellow

Dana-Farber Cancer Institute 